\title{
THE FREQUENCY OF HOT JUPITERS IN THE GALAXY: RESULTS FROM THE SuperLupus SURVEY
}

\author{
Daniel D. R. Bayliss and Penny D. Sackett \\ Research School of Astronomy and Astrophysics, The Australian National University, Mt Stromlo Observatory, \\ Cotter Road, Weston Creek, ACT 2611, Australia; daniel@mso.anu.edu.au \\ Received 2011 July 29; accepted 2011 October 18; published 2011 November 28
}

\begin{abstract}
We present the results of the SuperLupus Survey for transiting hot Jupiter planets, which monitored a single Galactic disk field spanning $0.66 \mathrm{deg}^{2}$ for 108 nights over three years. Ten candidates were detected: one is a transiting planet, two remain candidates, and seven have been subsequently identified as false positives. We construct a new image quality metric, $S_{j}$, based on the behavior of 26,859 light curves, which allows us to discard poor images in an objective and quantitative manner. Furthermore, in some cases we are able to identify statistical false positives by analyzing temporal correlations between $S_{j}$ and transit signatures. We use Monte Carlo simulations to measure our detection efficiency by injecting artificial transits onto real light curves and applying identical selection criteria as used in our survey. We find at $90 \%$ confidence level that $0.10_{-0.08}^{+0.27} \%$ of dwarf stars host a hot Jupiter with a period of 1-10 days. Our results are consistent with other transit surveys, but appear consistently lower than the hot Jupiter frequencies reported from radial velocity surveys, a difference we attribute, at least in part, to the difference in stellar populations probed. In light of our determination of the frequency of hot Jupiters in Galactic field stars, previous null results for transiting planets in open cluster and globular cluster surveys no longer appear anomalously low.
\end{abstract}

Key words: planets and satellites: detection - techniques: photometric

\section{INTRODUCTION}

The discovery of short-period, giant extrasolar planets (Mayor \& Queloz 1995; Marcy \& Butler 1996) provided the exciting potential for large numbers of planets to be discovered by the transit method, as these "hot Jupiters" have a $\sim 10 \%$ geometric probability of transiting, and do so every few days. However, early predictions greatly overestimated the actual discovery rate (Horne 2003). This discrepancy resulted from simplistic assumptions and a misunderstanding of the effects that systematic noise would play in lowering detection efficiency (Pont et al. 2006). It also resulted from an overestimation of the frequency of hot Jupiters. More accurate, and lower, predictions were provided in Beatty \& Gaudi (2008), where it was noted that objective and quantifiable detection criteria were required for more robust inferences of planet frequencies. By adopting such objective and quantifiable detection criteria, we propose that the frequency of hot Jupiters in the field has been overestimated by a factor of three (Bayliss \& Sackett 2011).

The SuperLupus Survey was established to detect hot Jupiters in a field positioned just above the Galactic plane $\left(b=11^{\circ}\right)$ and also to determine the fraction of stars that host hot Jupiters in this typical Galactic field. The survey design and data analysis were constructed so as to fulfill both of these objectives.

In Section 2 of this paper, we describe the SuperLupus observations and data reduction. The photometry is described in Section 3. The criteria for candidate selection are detailed in Section 4, and the 10 identified candidates are described and analyzed in Section 5.

In Section 6, we set out the details of the Monte Carlo simulations performed to calculate the detection efficiency and the effective number of stars probed for planets in the SuperLupus Survey. In Section 7, we apply this efficiency to the actual results of the survey to determine the fraction of stars in the field that host a hot Jupiter. Finally, in Section 8 we summarize and discuss the implications of our results.

\section{OBSERVATIONS AND DATA REDUCTION}

The SuperLupus Survey is an extension of the Lupus Survey (Bayliss et al. 2009), in which the duration of the original survey was approximately doubled to provide sensitivity to transiting planets with periods as long as 10 days. Many details of the data reduction procedure are set out elsewhere (Bayliss et al. 2009); here we summarize the procedure and highlight aspects that differed between the Lupus and SuperLupus projects.

Both surveys monitored a single field using the WideField Imager on the ANU 40 inch Telescope at Siding Spring Observatory. The field, in the constellation of Lupus, is $11^{\circ}$ above the Galactic plane centered at R.A. $=15^{\mathrm{h}} 30^{\mathrm{m}} 36^{\mathrm{s}} 3$, decl. $=-42^{\circ} 53^{\prime} 53^{\prime \prime} .0$ (J2000). In total, 5158 images of $300 \mathrm{~s}$ exposure were taken in a custom $(V+R)$ filter. Of these, 2801 images were from the original Lupus Survey (2005 and 2006) and an additional 2357 new images were taken in 2008. The average full width at half-maximum of the point-spread function (PSF) over all 5158 images is 2".02.

Initial data reduction (bias, dark, and flat-field correction) was carried out on the mosaic frames using standard IRAF ${ }^{1}$ tasks in the package MSCRED. Sky flats were obtained at twilight whenever conditions permitted, and master flat fields were produced by median combining the $\sim 30$ sky flats most proximate in time to each night. Bad pixels and columns were masked and the mosaic frames were split into individual CCD frames. A world coordinate system solution was calculated for each image in order to identify stars for photometry.

\section{PHOTOMETRY AND DETRENDING}

Photometry for the original Lupus Survey was performed using Difference Imaging Analysis (DIA; Alard \& Lupton 1998;

\footnotetext{
1 IRAF is distributed by the National Optical Astronomy Observatory, which is operated by the Association of Universities for Research in Astronomy, Inc., under cooperative agreement with the National Science Foundation.
} 
Wozniak 2000). For the SuperLupus Survey, we instead used Source Extractor (Bertin \& Arnouts 1996) aperture photometry. This approach was motivated by the fact that many of the survey images have PSFs that are asymmetric due to tracking or focusing issues. Such PSFs can cause potential problems for DIA photometry, but are not of concern for aperture photometry as long as the photometric aperture is large enough. Initial tests revealed that aperture photometry could achieve the same (and even better) photometric precision for the majority of the brighter stars $(V<20)$ in the field. In fact we note that the final SuperLupus aperture photometry resulted in approximately $10 \%$ more stars with a root-mean-squared (rms) variability less than 0.025 mag compared with the original Lupus Survey using DIA.

We found that a photometric aperture radius of 10 pixels (3.'75) resulted in the highest precision light curves for the majority of these stars.

A reference catalog of 50,907 target stars $(V<20)$ was produced from a single, high-quality, reference image. Aperture photometry was performed on these stars for each survey image, with apertures re-centered on the point of peak flux for each star in each image. Background subtraction was performed using a background map produced for each image by median filtering and bi-cubic-spline interpolation over a $8 \times 8$ pixel grid.

Systematic trends in the resulting light curves were removed using the Sys-Rem algorithm (Tamuz et al. 2005) with 12 iterations. To further refine this data set, we removed stars with light curves that displayed rms variability greater than $0.05 \mathrm{mag}$. We also discarded poor quality images as described below. In total this left us with 26,859 stars over 3585 images.

\subsection{Image Quality Metric}

To identify poor quality images, we define an image quality metric $\left(S_{j}\right)$ as

$$
S_{j}^{2}=\sum_{i} \frac{r_{i j}^{2}}{\sigma_{i j}^{2}},
$$

where for the $i$ th star on the $j$ th image, $r_{i j}$ is the average subtracted residual magnitude and $\sigma_{i j}$ is the photometric uncertainty. This metric is computed in the Sys-Rem algorithm, where linear systematic effects are removed in order to minimize $S_{j}$ (Tamuz et al. 2005). In essence, $S_{j}$ is a measure of how all the stars in image $j$ vary from the mean of all images in the survey. We retained only those images with $S_{j}<0.02$ for searching for planetary transits. The advantage of using this metric is that it uses information from all stars over the chip in a single quality factor that is directly related to the precision of the resulting stellar photometry.

This image quality metric also proved useful to determine if transit candidates were statistical false positives by looking for correlations between "transit" events and $S_{j}$ (see Section 5).

\section{TRANSIT SEARCH AND DETECTION CRITERIA}

In order to accurately determine the efficiency of the SuperLupus Survey, candidates must be identified in an automated and systematic manner that can be applied identically to synthetic light curves in the Monte Carlo simulation (see Section 6). This was implemented by way of a set of six selection criteria that were automatically applied to each processed light curve.

Initially all 26,859 SuperLupus light curves were searched for transit events using the Box-fitting Least-Squares (BLS) algorithm (Kovács et al. 2002). Trial periods in the range
Table 1

Candidate Selection Criteria

\begin{tabular}{lc}
\hline \hline Criterion & Threshold \\
\hline BLS signal detection efficiency & SDE $>7.0$ \\
Effective S/N of transit & $\alpha>10$ \\
BLS period & $P \neq 1.02$ days \\
& $P \neq 2.00 \pm 0.02$ days \\
Magnitude & $V<18.8$ \\
Single-event fraction & frac. $<0.9$ \\
Not a variable star & Weldrake \& Bayliss (2008) \\
& Catalog \\
\hline
\end{tabular}

1.01 days $<P<10$ days were tested with 55,000 equally spaced frequency steps and 200 phase bins per trial frequency. Candidates were then identified based on their BLS signal detection efficiency (SDE), as defined in Kovács et al. (2002). After testing synthetic light curves with the same temporal and noise characteristics as our data, a candidate threshold value of $\mathrm{SDE}=7.0$ was adopted.

We defined the effective signal-to-noise of the detected transit event, $\alpha$, for all candidates as

$$
\alpha=\frac{\delta}{\sigma} \sqrt{n_{\mathrm{obs}}},
$$

where $\delta$ is the BLS-determined transit depth, $\sigma$ is the rms variability of the entire (Sys-Rem-corrected) light curve, and $n_{\mathrm{obs}}$ is the actual number of data points within the BLSdetermined transit event, i.e., the number of "in-transit" data points. A threshold of $\alpha=10$ was adopted, identical to the threshold adopted in two other transit surveys of faint stars (Burke et al. 2006; Hartman et al. 2009). At this stage we also rejected candidates for which more than $90 \%$ of in-transit data originated from an event on a single night.

As with other ground-based surveys, many of our candidates displayed integer-day periods resulting from nightly systematic effects. Therefore, candidates with $1.01<P<1.02$ and $1.98<P<2.02$ were rejected. We also required that candidates had a magnitude of $V<18.8$.

Finally candidates were cross-matched with the catalog of known variable stars in the field (Weldrake \& Bayliss 2008) and eclipsing binary systems were removed.

The selection criteria are summarized in Table 1.

\section{INITIAL CANDIDATES}

A total of 10 light curves fulfilled the six selection criteria set out in Section 4. Their characteristics are described in Table 2.

The candidate SL-07 was immediately recognized as LupusTR-3, a star with a transiting planet that had been discovered during the original Lupus Survey (Weldrake et al. 2008; Bayliss et al. 2009). To investigate the likely nature of the other candidates, we used the $\eta_{p}$ diagnostic method proposed by Tingley $\&$ Sackett (2005). The results are set out in Table 3. Candidates SL-02 and SL-04 show very high diagnostic numbers, indicating that their transit parameters make them unlikely to be real transiting planets. In the case of candidate SL-04, a very clear transit was observed on 2005 June 6, with a transit time of approximately $6 \mathrm{hr}$, in contrast to the BLS-determined duration of $8.08 \mathrm{hr}$. Use of the shorter duration reduces the $\eta_{p}$ diagnostic value to 1.9 , which, while still high, could be explained by a large-radius planet, since the $\eta_{p}$ diagnostic is normalized to the assumption $R_{P}=1 R_{\mathrm{J}}$.

To help identify statistical false positives, we checked if the detected transit event was correlated with the image quality, 
Table 2

Properties of Initial SuperLupus Candidates

\begin{tabular}{|c|c|c|c|c|c|c|c|c|c|c|}
\hline ID & $\begin{array}{c}\text { R.A. } \\
\text { (J2000) }\end{array}$ & $\begin{array}{c}\text { Decl. } \\
(\mathrm{J} 2000)\end{array}$ & $\begin{array}{c}\text { Mag } \\
(V)\end{array}$ & $\begin{array}{l}\text { Period } \\
\text { (days) }\end{array}$ & $\begin{array}{c}\text { Depth } \\
(V+R)\end{array}$ & $\begin{array}{c}\text { Duration } \\
\text { (hr) }\end{array}$ & $\begin{array}{c}T_{C} \\
(\mathrm{HJD})\end{array}$ & SDE & $\alpha$ & $\eta_{p}$ \\
\hline SL-01 & $15: 30: 47.11$ & $-42: 44: 29.5$ & 16.8 & 8.59642 & 0.006 & 6.03 & 2454616.0322 & 7.53 & 11.4 & 1.2 \\
\hline SL-02 & $15: 31: 15.28$ & $-42: 47: 28.4$ & 16.7 & 7.44930 & 0.005 & 10.32 & 2453894.0654 & 8.00 & 13.1 & 2.1 \\
\hline SL-03 & $15: 30: 43.66$ & $-43: 01: 43.8$ & 18.2 & 5.38421 & 0.042 & 3.66 & 2453880.1516 & 8.60 & 21.0 & 1.4 \\
\hline SL-04 & $15: 31: 37.13$ & $-43: 10: 16.9$ & 17.8 & 7.72108 & 0.036 & 8.08 & 2453883.2487 & 8.30 & 32.4 & 1.9 \\
\hline SL-05 & $15: 30: 24.24$ & $-43: 15: 10.7$ & 18.4 & 1.88490 & 0.026 & 2.15 & 2453530.1826 & 7.70 & 16.2 & 1.0 \\
\hline SL-06 & 15:30:08.77 & $-43: 16: 04.5$ & 18.6 & 6.80834 & 0.039 & 1.92 & 2454615.9351 & 7.80 & 10.2 & 0.7 \\
\hline SL-07 & 15:30:18.71 & $-42: 58: 41.3$ & 17.6 & 3.91403 & 0.012 & 3.24 & 2453530.9068 & 9.05 & 13.4 & 1.0 \\
\hline SL-08 & $15: 28: 56.97$ & $-42: 55: 18.2$ & 18.2 & 7.08871 & 0.031 & 1.96 & 2454615.9576 & 9.13 & 11.4 & 0.6 \\
\hline SL-09 & $15: 29: 37.86$ & $-43: 07: 36.6$ & 18.4 & 5.88226 & 0.035 & 1.80 & 2454615.9640 & 7.53 & 12.0 & 0.6 \\
\hline SL-10 & $15: 29: 56.55$ & $-42: 33: 30.2$ & 17.9 & 5.12897 & 0.020 & 1.97 & 2454528.1707 & 7.70 & 10.8 & 0.6 \\
\hline
\end{tabular}

Table 3

Candidate Test Results

\begin{tabular}{lll}
\hline \hline ID & \multicolumn{1}{c}{ Discriminant } & \multicolumn{1}{c}{ Conclusion } \\
\hline SL-01 & Saturated close neighbor & Statistical false positive \\
SL-02 & High $\eta_{p}$ & EcB \\
SL-03 & Additional photometric analysis & Blended EcB \\
SL-04 & Transit depth $(0.042$ mag) suggests EcB & Candidate \\
SL-05 & Additional photometric analysis & EcB \\
SL-06 & Low $\alpha(10.2)$ & Candidate \\
SL-07 & Lupus-TR-3 & Planet \\
SL-08 & Transit correlated to $S_{j}$ & Statistical false positive \\
SL-09 & Transit correlated to $S_{j}$ & Statistical false positive \\
SL-10 & Transit correlated to $S_{j}$ & Statistical false positive \\
\end{tabular}

using the calculated value of the metric $S_{j}$, as defined in Equation (1).

Three candidates, SL-08, SL-09, and SL-10, were found to have transit events that were strongly correlated with $S_{j}$, indicating that the detected transit signature is almost certainly a systematic effect that the Sys-Rem algorithm had not fully removed. An example of such a correlation is shown in Figure 1.

We inspected the images of the candidates for signs that the transit signature might be caused by proximity to the edge of a $\mathrm{CCD}$, a bad column, or a bright star. We also inspected the image for evidence of significant flux contributions from neighboring stars within the photometric aperture.

SL-01 was found to be blended with a very bright, saturated neighboring star at a distance of $27^{\prime \prime}$. We therefore ruled this out as being a genuine candidate. We found that the candidate SL-03 had three neighbors close to the photometric aperture and that SL-05 had a neighbor well within the photometric aperture. Photometry for these candidates was re-derived using a smaller photometric aperture to avoid the contaminating neighbors. This analysis revealed that the transit event seen in SL-05 was in fact due to a very deep eclipse (40\%) occurring in a faint neighbor situated just 2".6 from SL-05. It also revealed that while the SL-03 target star was responsible for the observed transit event, close neighbors and the large aperture of the original photometry had diluted the size of the dip from the true depth of $0.100 \mathrm{mag}$ to a shallower $0.042 \mathrm{mag}$. The true depth is characteristic of an eclipsing binary and does not pass our selection criterion.

In summary, we conclude that of the 10 candidates identified in the SuperLupus Survey, only SL-07 can be confirmed as a genuine transiting planet. We rule out seven candidates (SL-01, SL-02, SL-03, SL-05, SL-08, SL-09, and SL-10) as being false positives, while two candidates (SL-04 and SL-06) remain. These two remaining candidates are $V=17.8$ and

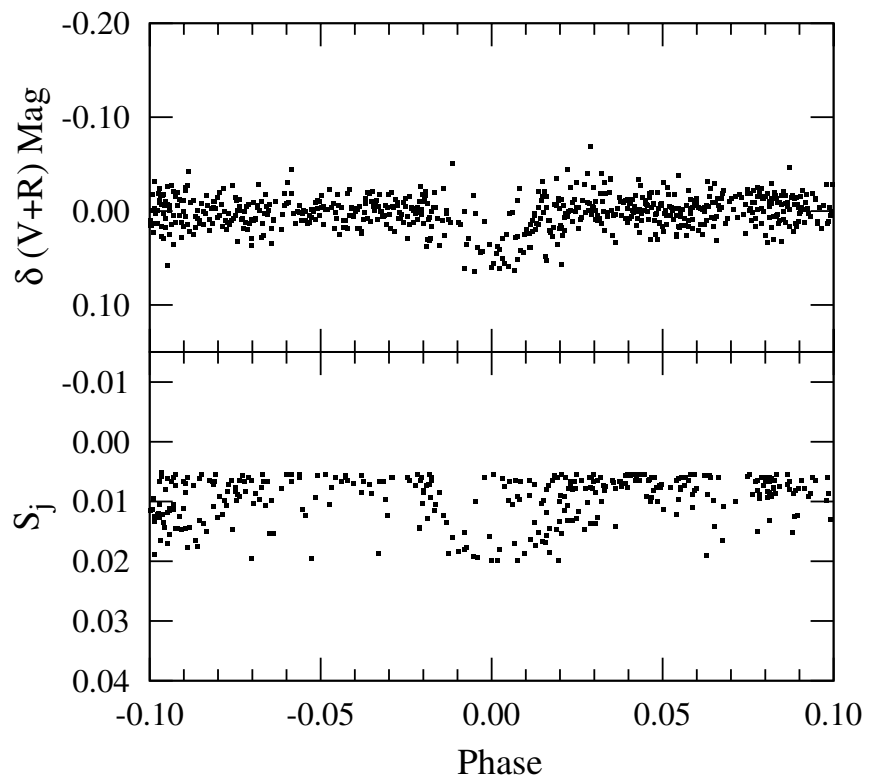

Figure 1. Phase-wrapped light curve for candidate SL-08 (top panel) and the corresponding image quality, $S_{j}$, for images with $S_{j}>0.005$ (bottom panel) wrapped at the same period and phase. The correlation between the transit event and image quality indicates that SL-08 is a statistical false positive.

$V=18.6$, respectively, making follow-up extremely difficult. Consequently, we have not observed these candidates further, although formally we cannot exclude them. In Section 7 we calculate hot Jupiter frequencies assuming only SL-07 is a transiting planet, but also provide figures for the case where one of these remaining candidates is a transiting planet.

\section{MONTE CARLO SIMULATIONS TO DETERMINE DETECTION EFFICIENCY}

To determine the efficiency of the SuperLupus Survey to detecting transiting planets, we carried out a Monte Carlo simulation of the SuperLupus Survey. Synthetic transit signatures were inserted into actual SuperLupus light curves and then recovery attempted using the same methodology and selection criteria used for our survey.

\subsection{Modeling the Stellar Population}

Generating a realistic set of synthetic transit light curves against which to test a detection algorithm requires knowledge of the actual distribution of transit depths, durations, and periods of transiting planets. Ideally, this would be achieved 


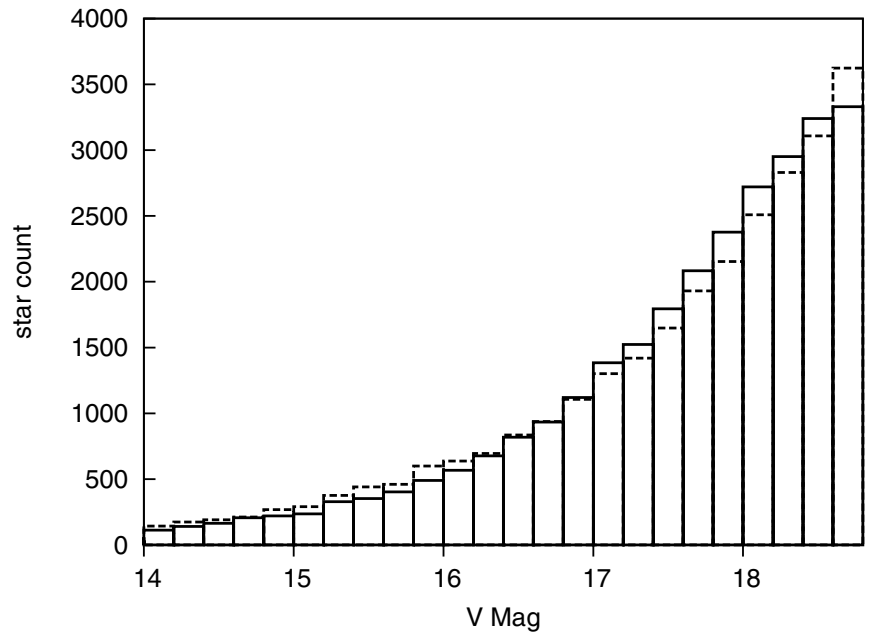

Figure 2. Comparison of the apparent $V$-magnitude distribution between the Beasonçon model (dashed line) and our observed field (solid line).

by determining the radius of each monitored star in the field. This is possible when monitoring an equidistant population such as an open cluster or a globular cluster (e.g., Hartman et al. 2009; Weldrake et al. 2005). Since the distances to field stars cannot be determined from photometry alone, the exact radius of each star in the SuperLupus field is unknown. We therefore used the Beasonçon model of the Galaxy (Robin et al. 2003) to provide a statistical distribution of stellar radii and masses for the stars monitored in our field. The population was synthesized over a $0.66 \mathrm{deg}^{2}$ field centered at $b=11^{\circ}, l=331^{\circ}$. Interstellar extinction was set at $0.6 \mathrm{mag} \mathrm{kpc}^{-1}$, based on the Schlegel dust maps of the region (Schlegel et al. 1998). Stars were selected in the magnitude range $14<V<18.8$ to match the SuperLupus observational and selection constraints. The Beasonçon model returned a population of stars that closely matched both the total star count and the distribution of apparent magnitudes (see Figure 2), giving us confidence this model accurately simulates our field.

We note that the Beasonçon model indicated that $24 \%$ of the survey stars have $\log g<4.0$. In our Monte Carlo simulation these stars are classified as giants and given zero probability for transit detection. We assign a radius to each star in the simulation based on the stellar mass from the Beasançon model and the simple relationship given by Cox (2000):

$$
\log \frac{R}{R_{\odot}}=0.917 \log \frac{M}{M_{\odot}}-0.020 .
$$

\subsection{Light Curve Generation}

Light curves for the Monte Carlo simulation were created by first randomly selecting a star from the Beasançon model population. The temporal sampling of the star is set to the actual timestamps of the SuperLupus Survey. We then need to attach an appropriate photometric uncertainty to each point on the synthetic curve.

This was done by using the $V$ magnitude of the chosen star to randomly select an rms uncertainty from the actual distributions in rms observed in the SuperLupus Survey for stars of that $V$ magnitude, as described below.

The rms scatter for each real light curve in the SuperLupus Survey, after the application of Sys-Rem, shows a wide range in rms scatter at a given $V$ magnitude. Therefore we calculated the actual distribution of rms scatter for each $V$ magnitude in the

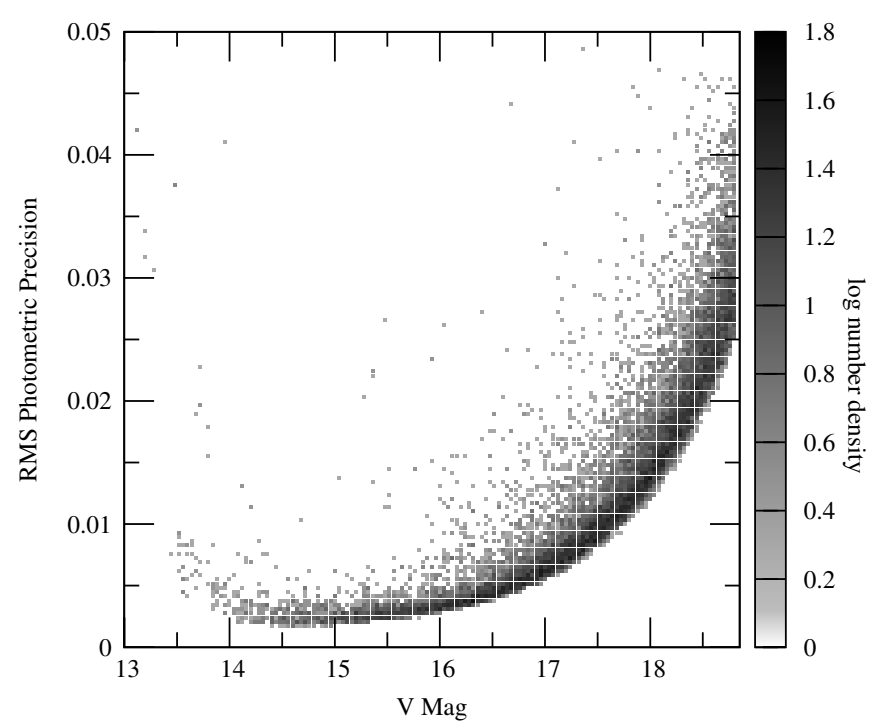

Figure 3. Number density of stars with a given rms photometric precision plotted against $V$ magnitude. The number density is given on a logarithmic scale. The bin width is 0.04 mag for $V$ magnitude and 0.3 mmag for rms photometric precision.

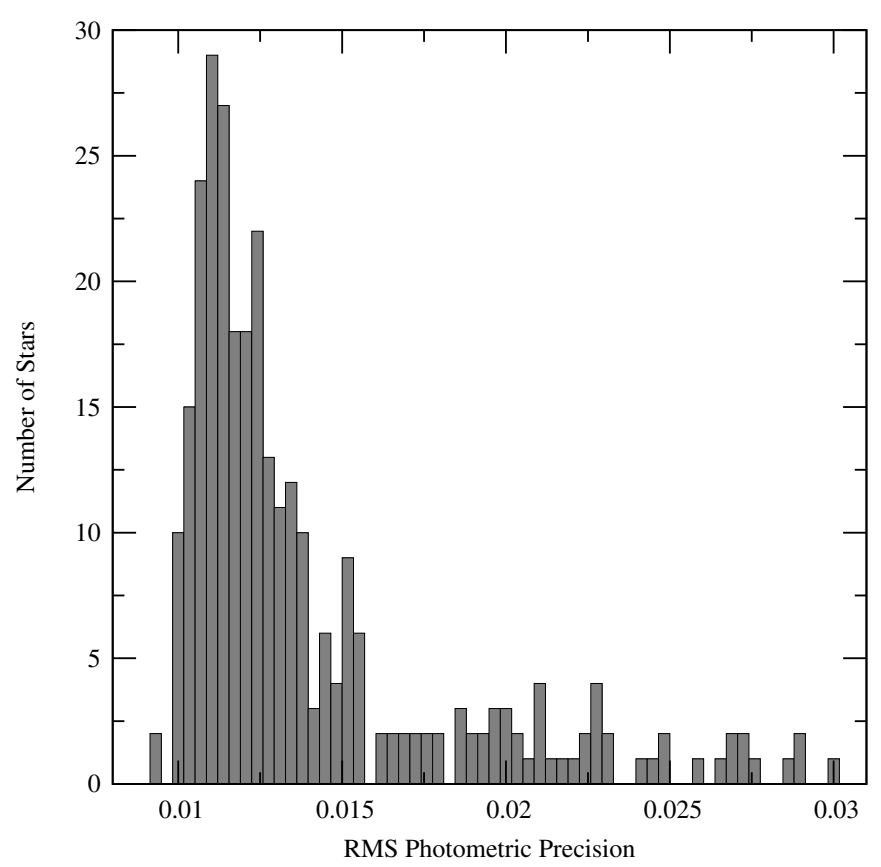

Figure 4. rms photometric precision distribution profile for stars in the SuperLupus Survey between $V=17.70$ and $V=17.74$.

survey, as displayed in Figure 3. As an example, the distribution for $V=17.72$ is shown in Figure 4. It can be thought of as a vertical slice through the density plot in Figure 3.

It is evident that the rms scatter distributions are nonGaussian, with long tails toward high rms scatter. This further highlights the importance of basing the rms scatter of synthetic light curves on the actual distributions of photometric precision rather than approximating them using a single-valued function of magnitude as has been done in some previous studies (e.g., Weldrake et al. 2005; Gould et al. 2006).

A value for the photometric precision is selected randomly, using the appropriate rms scatter distribution as a probability weighting, for each Beasonçon star. Once the value of the photometric precision is determined, a real SuperLupus light 
curve is selected with the same photometric precision, and used as the base light curve into which we inject a transit.

\subsection{Injecting Transits}

To simulate the light curve from a transiting hot Jupiter, we first determined the parameters for each simulated system. The stellar mass and radius were taken from the Beasonçon model and Equation (3). The planetary mass was fixed at $1 M_{\mathrm{J}}$, but we note that this does not significantly alter the transit parameters when $M_{P} \ll M_{\star}$. Four different planetary radii were simulated: $R_{P}=0.8,1.0,1.2$, and $1.4 R_{\mathrm{J}}$.

The inclination of the orbit, $i$, was determined by choosing a random $\cos i$ between zero and $\cos i_{\min }$, the limiting inclination that results in a transit (Sackett 1999):

$$
\cos i_{\min }=\frac{R_{\star}+R_{P}}{a},
$$

where $a$ is the planet's semimajor orbital axis.

Although there is some evidence for a "pile-up" of planets at $\sim 3$ day periods (Cumming et al. 2008), the intrinsic distribution of planets with periods between 1 and 10 days is not well understood. Therefore, it is necessary to make an assumption in assigning the period distribution in the Monte Carlo simulation. Hartman et al. (2009) use a uniform logarithmic distribution. We test this distribution as well as a uniform linear distribution in period.

With the parameters $M_{\star}, M_{P}, R_{\star}, R_{P}, \cos i$, and $P$ assigned to each star, a transit depth and duration were calculated and a boxshaped transit inserted into each synthetic light curve. For each parameter set, we simulated 10 randomly selected phases. We adopted a simple box-shaped model as limb-darkening effects have been shown to be negligible in this signal-to-noise regime (Gould et al. 2006). The transits were inserted into light curves that had already been detrended using Sys-Rem, whereas a transit signal in our survey would need to pass through the SysRem detrending. We therefore carefully tested and optimized the number of Sys-Rem iterations we used so that we would not degrade a real astrophysical signal. Only those trends that are present in many light curves with the same temporal timestamps are removed.

\subsection{Detection Efficiency and Effective Number of Stars Probed}

The BLS algorithm (Kovács et al. 2002) was applied to each synthetic light curve, using the same parameters used for the actual SuperLupus data set (see Section 4). In total, 800,000 light curves were generated for the Monte Carlo simulation and searched for transit signatures. Transits were deemed to be detected if the selection criteria used in the actual SuperLupus search (see Section 4) were satisfied.

The detection efficiency, $\varepsilon$, is defined as the fraction of light curves in which the inserted transit signal is detected in the Monte Carlo simulation:

$$
\varepsilon=\frac{1}{N_{\text {sim }}} \sum_{i=1}^{N_{\text {sim }}} \delta_{i,},
$$

where $\delta_{i}$ is a delta function representing whether the transit of star $i$ is detected $\left(\delta_{i}=1\right)$ or not detected $\left(\delta_{i}=0\right)$, and $N_{\text {sim }}$ is the number of simulated stars in the Monte Carlo simulation. As discussed in Section $6.1,24 \%$ of our survey stars are giants $(\log g<4.0)$ and were automatically assigned a $\delta_{i}=0$ regardless of the other system parameters. We also
Table 4

\begin{tabular}{|c|c|c|c|c|c|c|}
\hline $\begin{array}{l}\text { Period } \\
\text { Range and } \\
\text { Distribution }^{\mathrm{a}}\end{array}$ & $\begin{array}{c}\text { Planet } \\
\text { Radius } \\
\left(R_{\mathrm{J}}\right)\end{array}$ & $\begin{array}{l}\text { Mean } \\
\text { Depth } \\
\text { (mag) }\end{array}$ & $\begin{array}{c}\text { Mean } \\
\text { Duration } \\
\text { (hr) }\end{array}$ & $\begin{array}{c}\text { Mean } \\
\text { Probability } \\
\text { of Transit }\end{array}$ & $\begin{array}{l}\text { Detection } \\
\text { Efficiency } \\
\quad(\varepsilon)\end{array}$ & $\begin{array}{c}\text { Stars } \\
\text { Probed } \\
\left(N_{\mathrm{pr}}\right)\end{array}$ \\
\hline \multirow[t]{4}{*}{ 1-10 days, Uni } & 0.8 & 0.010 & 2.44 & 0.091 & 0.12 & 231 \\
\hline & 1.0 & 0.016 & 2.49 & 0.093 & 0.27 & 488 \\
\hline & 1.2 & 0.023 & 2.53 & 0.095 & 0.43 & 762 \\
\hline & 1.4 & 0.031 & 2.60 & 0.097 & 0.55 & 967 \\
\hline \multirow[t]{4}{*}{ 1-3 days, Uni } & 0.8 & 0.010 & 1.80 & 0.156 & 0.28 & 697 \\
\hline & 1.0 & 0.016 & 1.81 & 0.161 & 0.51 & 1311 \\
\hline & 1.2 & 0.023 & 1.84 & 0.164 & 0.73 & 1890 \\
\hline & 1.4 & 0.031 & 1.89 & 0.167 & 0.85 & 2219 \\
\hline \multirow[t]{4}{*}{ 3-5 days, Uni } & 0.8 & 0.010 & 2.26 & 0.095 & 0.15 & 228 \\
\hline & 1.0 & 0.016 & 2.30 & 0.097 & 0.34 & 527 \\
\hline & 1.2 & 0.022 & 2.35 & 0.099 & 0.54 & 851 \\
\hline & 1.4 & 0.031 & 2.40 & 0.101 & 0.71 & 1127 \\
\hline \multirow[t]{4}{*}{ 5-10 days, Uni } & 0.8 & 0.010 & 2.78 & 0.063 & 0.05 & 46 \\
\hline & 1.0 & 0.016 & 2.84 & 0.064 & 0.13 & 141 \\
\hline & 1.2 & 0.023 & 2.89 & 0.066 & 0.26 & 275 \\
\hline & 1.4 & 0.031 & 2.96 & 0.067 & 0.36 & 392 \\
\hline \multirow[t]{4}{*}{ 1-10 days, Log } & 0.8 & 0.010 & 2.15 & 0.120 & 0.18 & 416 \\
\hline & 1.0 & 0.016 & 2.20 & 0.122 & 0.37 & 841 \\
\hline & 1.2 & 0.023 & 2.23 & 0.125 & 0.54 & 1216 \\
\hline & 1.4 & 0.031 & 2.28 & 0.128 & 0.68 & 1517 \\
\hline \multirow[t]{4}{*}{ 1-3 days, Log } & 0.8 & 0.010 & 1.72 & 0.168 & 0.28 & 759 \\
\hline & 1.0 & 0.016 & 1.77 & 0.171 & 0.54 & 1466 \\
\hline & 1.2 & 0.023 & 1.79 & 0.174 & 0.73 & 2007 \\
\hline & 1.4 & 0.031 & 1.83 & 0.178 & 0.85 & 2391 \\
\hline \multirow[t]{4}{*}{ 3-5 days, Log } & 0.8 & 0.010 & 2.24 & 0.096 & 0.13 & 193 \\
\hline & 1.0 & 0.015 & 2.25 & 0.098 & 0.32 & 502 \\
\hline & 1.2 & 0.022 & 2.34 & 0.101 & 0.53 & 841 \\
\hline & 1.4 & 0.031 & 2.36 & 0.102 & 0.70 & 1124 \\
\hline \multirow[t]{4}{*}{ 5-10 days, Log } & 0.8 & 0.010 & 2.76 & 0.065 & 0.05 & 50 \\
\hline & 1.0 & 0.016 & 2.79 & 0.066 & 0.14 & 152 \\
\hline & 1.2 & 0.023 & 2.83 & 0.068 & 0.26 & 281 \\
\hline & 1.4 & 0.031 & 2.92 & 0.069 & 0.38 & 431 \\
\hline
\end{tabular}

Monte Carlo Results

Note. ${ }^{a}$ Uni: uniform distribution; Log: logarithmic distribution.

note that only transiting systems were simulated, so $\varepsilon$ does not include the geometric probability of transit.

To determine the frequency of hot Jupiters in the field of the SuperLupus Survey we need to determine how many dwarf stars we have effectively probed for transiting planets, $N_{\mathrm{pr}}$. This number depends on the geometric probability of transit, the number of stars monitored to a given precision (for SuperLupus this is 20,465), as well as the detection efficiency. We calculated the geometric probability of transit, $f_{i}^{\text {geo }}$, for each system generated in our Monte Carlo simulation. $N_{\mathrm{pr}}$ is therefore given as

$$
N_{\mathrm{pr}}=\frac{N_{\mathrm{sur}}}{N_{\mathrm{sim}}} \sum_{i=1}^{N_{\mathrm{sim}}} \delta_{i} f_{i}^{\mathrm{geo}}
$$

Both the detection efficiency and the effective number of dwarf stars probed are set out in Table 4 for a variety of period ranges and assumed period distributions of hot Jupiters. Also tabulated are the mean transit depth, transit duration, and mean transit probability for each set of Monte Carlo assumptions about the size and period distribution of hot Jupiters. The results are presented for all periods simulated (1-10 days) as well as for period ranges of 1-3 days, 3-5 days, and 5-10 days in order 
Table 5

Frequency of Hot Jupiters

\begin{tabular}{|c|c|c|c|c|c|c|}
\hline \multirow{2}{*}{$\begin{array}{l}\text { Period } \\
\text { Range and } \\
\text { Distribution }^{\mathrm{a}}\end{array}$} & \multirow{2}{*}{$\begin{array}{c}\text { Planets } \\
\text { Detected }^{\mathrm{b}} \\
\quad(n)\end{array}$} & \multicolumn{5}{|c|}{ Planet Frequency $(f)$} \\
\hline & & $0.8 R_{\mathrm{J}}$ & $1.0 R_{\mathrm{J}}$ & $1.2 R_{\mathrm{J}}$ & $1.4 R_{\mathrm{J}}$ & Mean $R_{P}$ \\
\hline 1-10 days, Uni & $\begin{array}{c}1 \\
{[2]}\end{array}$ & $0.43_{-0.28}^{+1.62} \%$ & $\begin{array}{l}<0.62 \% \\
{\left[0.20_{-0.13}^{+0.77} \%\right]}\end{array}$ & $<0.39 \%$ & $<0.31 \%$ & $\begin{array}{c}0.16_{-0.10}^{+0.62} \% \\
{\left[0.32_{-0.19}^{+0.70} \%\right]}\end{array}$ \\
\hline 1-3 days, Uni & 0 & $<0.43 \%$ & $<0.23 \%$ & $<0.16 \%$ & $<0.14 \%$ & $<0.20 \%$ \\
\hline 3-5 days, Uni & 1 & $0.44_{-0.28}^{+1.65} \%$ & $<0.57 \%$ & $<0.35 \%$ & $<0.27 \%$ & $0.15_{-0.10}^{+0.54} \%$ \\
\hline 5-10 days, Uni & $\begin{array}{c}0 \\
{[1]}\end{array}$ & $<6.53 \%$ & $\begin{array}{l}<2.13 \% \\
{\left[0.71_{-0.46}^{+2.66} \%\right]}\end{array}$ & $<1.09 \%$ & $<0.77 \%$ & $\begin{array}{l}<1.41 \% \\
{\left[0.47_{-0.30}^{+1.75} \%\right]}\end{array}$ \\
\hline $1-10$ days, $\log$ & $\begin{array}{c}1 \\
{[2]}\end{array}$ & $0.24_{-0.18}^{+0.65 \%}$ & $\begin{array}{l}<0.25 \% \\
\left(0.12_{-0.09}^{+0.32} \%\right)\end{array}$ & $<0.18 \%$ & $<0.14 \%$ & $\begin{array}{c}0.10_{-0.08}^{+0.27} \% \\
\left(0.20_{-0.14}^{+0.32} \%\right)\end{array}$ \\
\hline 1-3 days, $\log$ & 0 & $<0.28 \%$ & $<0.15 \%$ & $<0.11 \%$ & $<0.09 \%$ & $<0.13 \%$ \\
\hline 3-5 days, $\log$ & 1 & $0.52_{-0.40}^{+1.40} \%$ & $<0.43 \%$ & $<0.25 \%$ & $<0.19 \%$ & $0.15_{-0.11}^{+0.41} \%$ \\
\hline 5-10 days, $\log$ & $\begin{array}{c}0 \\
{[1]}\end{array}$ & $<4.27 \%$ & $\begin{array}{l}<1.41 \% \\
{\left[0.66_{-0.50}^{+1.78} \%\right]}\end{array}$ & $<0.76 \%$ & $<0.50 \%$ & $\begin{array}{l}<0.93 \% \\
{\left[0.44_{-0.34}^{+1.19} \%\right]}\end{array}$ \\
\hline
\end{tabular}

Notes.

${ }^{a}$ Uni: uniform distribution; Log: logarithmic distribution.

${ }^{\mathrm{b}}$ Statistics in square brackets are for cases where either SL-04 or SL-06 is a planet.

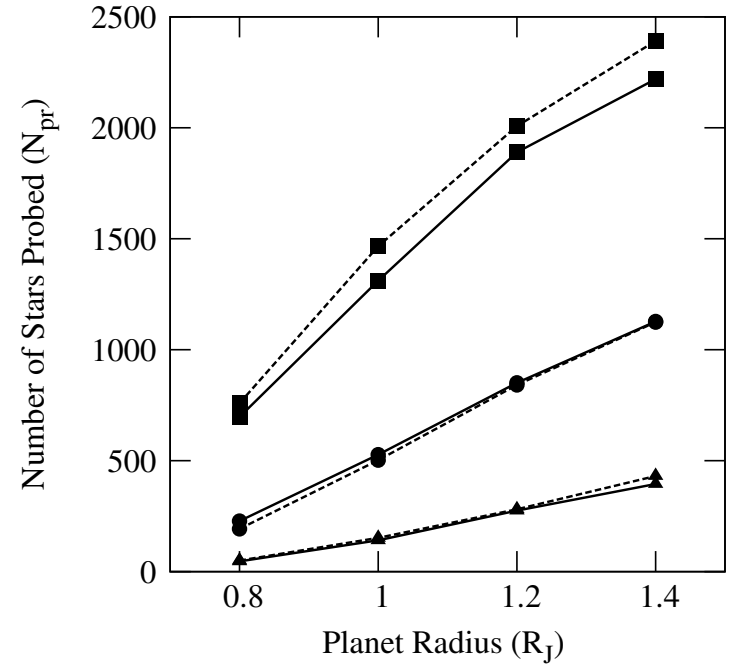

Figure 5. Effective number of dwarf stars probed $\left(N_{\mathrm{pr}}\right)$ for transiting hot Jupiters in the SuperLupus Survey as a function of planet radius. The solid lines represent the assumption of a uniform period distribution, while the dashed lines represent the assumption of a log uniform distribution. The square symbols are for 1-3 day periods, the circles for 3-5 day periods, and the triangles for 5-10 day periods.

to allow for direct comparison with the results of Gould et al. (2006) and Hartman et al. (2009).

In Figure $5, N_{\mathrm{pr}}$ is plotted as a function of planet radius for each of the four planetary radii simulated. As expected, more stars are probed in all period ranges for large-radii planets and more stars are probed for shorter period planets. The difference between the uniform and uniform logarithmic period distributions is most marked for the 1-3 day period planets.

\section{FREQUENCY OF PLANETS IN THE SURVEY}

The frequency of dwarf stars that host hot Jupiter planets, $f$, can be calculated simply using the Monte Carlo results and the
SuperLupus Survey results:

$$
f=\frac{n}{N_{p r}}
$$

where $n$ is the number of planets detected in the survey.

With only one (or possibly two) planets detected, Poisson statistics are used to determine confidence intervals or upper limits in the cases of no planet detections. The probability of detecting $n$ planets when $\lambda$ planets are expected is

$$
P(n, \lambda)=e^{\lambda} \frac{\lambda^{n}}{n !}
$$

Since the underlying distribution of planets is unknown, a Bayesian approach is used, with a flat prior for the uniform distribution, and a log prior for the logarithmic distribution. The upper and lower $90 \%$ confidence intervals ( $\sigma_{\text {high }}$ and $\sigma_{\text {low }}$, respectively) are calculated by solving

$$
\frac{\int_{\sigma_{\text {low }}}^{\sigma_{\text {high }}} \frac{e^{\lambda} \lambda^{n}}{n !} d \lambda}{\int_{0}^{\infty} \frac{e^{\lambda} \lambda^{n}}{n !} d \lambda}=0.9 .
$$

The values of $\sigma_{\text {high }}$ and $\sigma_{\text {low }}$ were determined numerically from Equation (9), with a symmetric confidence interval such that $5 \%$ of the probability distribution was below $\sigma_{\text {low }}$ and $5 \%$ above $\sigma_{\text {high }}$. The limits $\sigma_{\text {high }}$ and $\sigma_{\text {low }}$ were then used to calculate the upper and lower limits for $90 \%$ confidence intervals.

Where no planets were detected in a period range, a one-sided $95 \%$ confidence upper limit is used so that it can be directly compared with the two-sided $90 \%$ limits. In that case, $n=0$ and Equation (9) simplifies to

$$
1-e^{-\sigma_{\text {high }}}=0.95 \text {. }
$$

This method of calculating confidence limits is similar to that used by Gould et al. (2006) and Hartman et al. (2009).

The follow-up work presented in Weldrake et al. (2008) reveals that one of the SuperLupus candidates, SL-07, is indeed 
Table 6

Comparison of Planet Frequencies

\begin{tabular}{|c|c|c|c|}
\hline $\begin{array}{l}\text { Survey and } \\
\text { Reference }\end{array}$ & $\begin{array}{l}\text { Method and } \\
\text { Population }\end{array}$ & Assumptions & $\begin{array}{c}\text { Planet } \\
\text { Frequency }\end{array}$ \\
\hline $\begin{array}{l}\text { Kepler } \\
\text { (Howard et al. 2011) }\end{array}$ & $\begin{array}{l}\text { Transit, } \\
\text { "solar-type" } \\
\text { dwarf stars }\end{array}$ & $\begin{array}{l}1.2 \text { days }<P<10 \text { days } \\
0.7 R_{\mathrm{J}}<R_{P}<1.4 R_{\mathrm{J}} \\
n=22\end{array}$ & $0.37 \%$ \\
\hline $\begin{array}{l}\text { Keck Planet Search } \\
\text { (Cumming et al. 2008) }\end{array}$ & $\begin{array}{l}\text { Radial velocity, } \\
\text { nearby dwarf stars }\end{array}$ & $\begin{array}{l}M_{P}>0.5 M_{\mathrm{J}} \\
P<5 \text { days }\end{array}$ & $0.65 \pm 0.4 \%$ \\
\hline $\begin{array}{l}\text { ELODIE Planet Search } \\
\text { (Naef et al. 2005) }\end{array}$ & $\begin{array}{l}\text { Radial velocity, } \\
\text { nearby dwarf stars }\end{array}$ & $\begin{array}{l}M_{P}>0.5 M_{\mathrm{J}} \\
P<5 \text { days }\end{array}$ & $0.7 \pm 0.5 \%$ \\
\hline $\begin{array}{l}\text { SWEEPS } \\
\text { (Sahu et al. 2006) }\end{array}$ & $\begin{array}{l}\text { Transit, } \\
\text { bulge stars, } \\
M_{\star}>0.5 M_{\odot}\end{array}$ & $\begin{array}{l}P<4.2 \text { days } \\
n=16\end{array}$ & $0.4_{-0.2}^{+0.4} \%$ \\
\hline $\begin{array}{l}\text { OGLE-III } \\
\text { (Gould et al. 2006) }\end{array}$ & $\begin{array}{l}\text { Transit, } \\
\text { bulge and Galactic } \\
\text { disk dwarfs stars }\end{array}$ & $\begin{array}{l}n=5,1.0 R_{\mathrm{J}}<R_{P}<1.25 R_{\mathrm{J}} \\
\log P \text { distribution, } \\
1 \text { day } \leqslant P \leqslant 3 \text { days } \\
3 \text { days } \leqslant P \leqslant 5 \text { days }\end{array}$ & $\begin{array}{l}0.14_{-0.08}^{+0.15} \% \\
0.31_{-0.18}^{+0.42} \%\end{array}$ \\
\hline $\begin{array}{l}\text { Deep MMT } \\
\text { (Hartman et al. 2009) }\end{array}$ & $\begin{array}{l}\text { Transit, } \\
\text { Galactic disk } \\
\text { dwarf stars }\end{array}$ & $\begin{array}{l}n=0, R=1.0 R_{\mathrm{J}} \\
\log P \text { distribution } \\
0.4 \text { days } \leqslant P \leqslant 1 \text { days } \\
1 \text { day } \leqslant P \leqslant 3 \text { days } \\
3 \text { days } \leqslant P \leqslant 5 \text { days }\end{array}$ & $\begin{array}{l}<0.3 \% \\
<0.8 \% \\
<2.7 \%\end{array}$ \\
\hline $\begin{array}{l}\text { SuperLupus } \\
\text { (This Work) }\end{array}$ & $\begin{array}{l}\text { Transit, } \\
\text { Galactic disk } \\
\text { dwarf stars }\end{array}$ & $\begin{array}{l}n=1, R=1.1 R_{\mathbf{J}} \\
\log P \text { distribution } \\
n=1 \text { (Lupus-TR-3b) } \\
1 \text { day } \leqslant P \leqslant 3 \text { days } \\
3 \text { days } \leqslant P \leqslant 5 \text { days } \\
5 \text { days } \leqslant P \leqslant 10 \text { days } \\
1 \text { day } \leqslant P \leqslant 10 \text { days }\end{array}$ & $\begin{array}{c}<0.15 \% \\
0.15_{-0.11}^{+0.41} \% \\
<0.93 \% \\
0.10_{-0.08}^{+0.27 \%}\end{array}$ \\
\hline
\end{tabular}

a hot Jupiter planet. Seven other candidates can be ruled out as false positives, while SL-04 and SL-06 remain as candidates, although follow-up would be difficult due to their faintness. We therefore present our frequency results for the case where LupusTR-3b is the only transiting planet detected $(n=1)$ and also for the case where either SL-04 or SL-06 is also a transiting planet $(n=2)$. The results are set out in Table 5, again for both uniform and uniform logarithmic period distributions, and are tabulated in the same period ranges as used in Table 4. Frequencies for each of the four simulated planet radii are calculated, along with the mean over these radii, which is $R_{P}=1.1$.

\subsection{Comparison with Other Surveys}

There are four other transit studies of non-cluster fields that report statistics for hot Jupiter frequency.

The Kepler Mission (Borucki et al. 1997) yields very high precision, near continuous photometry for around 150,000 target stars in a selected Galactic field. Howard et al. (2011) calculate the frequency of planets within $0.25 \mathrm{AU}$ of solar-type host stars selected from the Kepler target stars. For the period range of 1.2 days $<P<10$ days, and radii $0.7<R_{\mathrm{J}}<1.4$, very similar to those considered in this work, the frequency of hot Jupiters is given as $0.37 \%$ (Figure 4 in Howard et al. 2011). This frequency is based only on "solar-type" Kepler target stars, defined as those with temperatures of $T_{\text {eff }}=4100-6100 \mathrm{~K}$ and gravities of $4.0<\log g<4.9$. While this gravity cut is essentially the same as is used in this work, the temperature cut means that a more limited sample of stars are being probed. Coupled with the fact that these stars are already drawn from a set of targets selected using "detectability metrics" that suggested they were most likely to give a detectable transit signal for terrestrial-size planets (Batalha et al. 2010), the frequency of $0.37 \%$ is higher than one might expect for a ground-based survey of random Galactic dwarfs in the field. Indeed the Kepler result is at the high end of the frequency of hot Jupiters determined from our SuperLupus survey, just consistent within our uncertainty.

The MMT transit survey of the open cluster M37 (Hartman et al. 2009) included a significant number of non-cluster field stars, and these were analyzed separately to determine the hot Jupiter frequency for Galactic dwarf stars. Assuming a Jupitersized planet, and a logarithmic period distribution, that study found that the fraction of field stars hosting planets with periods of 1-3 days is $<0.8 \%$, and those with planets having periods of $3-5$ days is $<2.7 \%$. These statistics are upper limits only, as there were no confirmed transiting planets.

Analysis of the Optical Gravitational Lensing Experiment III (OGLE-III) survey for transiting planets puts the frequency of hot Jupiters at $0.14 \%$ for $1-3$ day period planets, and $0.31 \%$ for periods of 3-5 days (Gould et al. 2006). These statistics draw on survey fields in both the Galactic plane and the Galactic bulge, and were derived based on five transiting planets that had been confirmed from the OGLE-III survey. They rely on an estimation of the efficiency of the "by-eye" selection that was used by the OGLE team to select transiting planet candidates.

The Hubble Space Telescope was used to undertake a survey for transiting planets in the crowded bulge region of the Galaxy (Sahu et al. 2006). Sixteen candidates were discovered from a sample of 180,000 stars monitored; however only one of these (SWEEPS-11) was bright enough to be confirmed in the usual 
manner using radial velocity (RV) measurements. Assuming that all the candidates are indeed planets, it was reported that $0.42 \%$ of bulge stars more massive than $0.44 M_{\odot}$ have giant planets with periods up to 4.2 days (Sahu et al. 2006).

Our result for $1-3$ day period planets, $f<0.13 \%$, is consistent with the limits from the deep MMT survey of Hartman et al. (2009). It is lower than the OGLE results, but consistent within the lower half of their $90 \%$ confidence interval. For 3-5 day period planets, our result of $f=0.15_{-0.11}^{+0.41}$ is consistent with the upper limit from the deep MMT survey. It is lower than the OGLE results, but still within the $90 \%$ confidence intervals. For 5-10 day periods we are able to place limits on the frequency of hot Jupiters of $f<0.93 \%$, assuming a logarithmic distribution in period and also that SL-04 and SL-06 do not host genuine hot Jupiters. If one of these candidates is a transiting hot Jupiter this frequency in this period range would be $0.44_{-0.34}^{+1.19} \%$, closer to the Kepler figure of $0.37 \%$ but still lower than the RV survey results. The comparisons are summarized in Table 6.

The frequency of hot Jupiters can also be determined from RV surveys. Cumming et al. (2008) report a frequency of $0.65 \pm 0.4 \%$ from the Keck Planet Search, while Naef et al. (2005) report a frequency of $0.7 \pm 0.5 \%$ from the ELODIE Planet Search. These comparisons are also set out in Table 6. Both of these RV results are above our upper $90 \%$ confidence interval $(0.56 \%)$, although the confidence intervals of the two survey results overlap.

There are, however, major differences between transit surveys and these RV surveys that could lead to discrepancies in the frequency derived for hot Jupiters. RV surveys are sensitive to planetary mass, while transit surveys are sensitive to planetary radius. Also transit and RV surveys usually monitor different stellar populations. RV surveys typically monitor carefully selected bright, sun-like stars. More recently, bright $\mathrm{M}$ dwarfs have also been monitored (Forveille et al. 2009). Transit searches monitor every star in the survey field that has the requisite signalto-noise to yield high-precision photometry. In a deep survey such as the SuperLupus Survey, this will translate to a stellar population with a sub-solar mean mass $\left(0.9 M_{\odot}\right.$ in the case of the SuperLupus Survey). If hot Jupiters of a given period are less frequent around lower mass stars, then the frequency of hot Jupiters from deep transit surveys will be lower than RV surveys.

\section{DISCUSSION}

The frequency of hot Jupiters in the Galaxy is an important quantity that will ultimately provide a constraint on models of planet formation and migration.

It has been suggested that the frequency of hot Jupiters in globular cluster environments is lower than that of field stars and that this may be due to crowding or low metallicity affecting planet formation, migration, or survival (Gilliland et al. 2000; Weldrake et al. 2005). Our results indicate, however, that there is little statistical disagreement between hot Jupiter frequencies in cluster and non-cluster environments, even if one of the remaining candidates turns out to be a genuine planet. This is consistent with the work presented in Saders \& Gaudi (2011) which concludes that there is no evidence to support that open clusters have a lower frequency of hot Jupiters.
Initial estimates for planet yields from transit surveys turned out to be far in excess of the actual discovery rate (Horne 2003). One of the many factors that led to this overestimation was the adoption of the hot Jupiter frequency derived from early RV surveys, which as we have shown is higher than is found from transit surveys. The Kepler result of $0.37 \%$ (Howard et al. 2011) obviously provides a robust statistic for hot Jupiter frequencies due to the high detection efficiency of that survey. However the result should be adopted cautiously when calculating expected yields from typical transit surveys, as it is based on a sample of "solar-type" stars drawn from Kepler target stars, rather than the ensemble field stars monitored by most blind transit surveys.

The authors thank David Weldrake and Brandon Tingley, who initiated the original Lupus Survey with P.D.S. We also thank Grant Kennedy and Tom Evans for assisting in gathering data for the SuperLupus project.

Facility: SSO:1m (WFI)

\section{REFERENCES}

Alard, C., \& Lupton, R. H. 1998, ApJ, 503, 325

Batalha, N. M., Borucki, W. J., Koch, D. G., et al. 2010, ApJ, 713, L109

Bayliss, D. D. R., \& Sackett, P. D. 2011, in Detection and Dynamics of Transiting Exoplanets, ed. F. Bouchy, R. Diaz, \& C. Moutou, EPJ Web of Conferences, 11,01008

Bayliss, D. D. R., Weldrake, D. T. F., Sackett, P. D., Tingley, B. W., \& Lewis, K. M. 2009, AJ, 137, 4368

Beatty, T. G., \& Gaudi, B. S. 2008, ApJ, 686, 1302

Bertin, E., \& Arnouts, S. 1996, A\&AS, 117, 393

Borucki, W. J., Koch, D. G., Dunham, E. W., \& Jenkins, J. M. 1997, in ASP Conf. Ser. 119, Planets Beyond the Solar System and the Next Generation of Space Missions, ed. D. Soderblom (San Francisco, CA: ASP), 153

Burke, C. J., Gaudi, B. S., DePoy, D. L., \& Pogge, R. W. 2006, AJ, 132, 210

Cox, A. N. (ed.) 2000, Allen's Astrophysical Quantities (4th ed.; New York: AIP Press)

Cumming, A., Butler, R. P., Marcy, G. W., et al. 2008, PASP, 120, 531

Forveille, T., Bonfils, X., Delfosse, X., et al. 2009, A\&A, 493, 645

Gilliland, R. L., Brown, T. M., Guhathakurta, P., et al. 2000, ApJ, 545, L47

Gould, A., Dorsher, S., Gaudi, B. S., \& Udalski, A. 2006, Acta Astron., 56, 1

Hartman, J. D., Gaudi, B. S., Holman, M. J., et al. 2009, ApJ, 695, 336

Horne, K. 2003, in ASP Conf. Ser. 294, Scientific Frontiers in Research on Extrasolar Planets, ed. D. Deming \& S. Seager (San Francisco, CA: ASP), 361

Howard, A. W., Marcy, G. W., Bryson, S. T., et al. 2011, arXiv:1103.2541

Kovács, G., Zucker, S., \& Mazeh, T. 2002, A\&A, 391, 369

Marcy, G. W., \& Butler, R. P. 1996, ApJ, 464, L147

Mayor, M., \& Queloz, D. 1995, Nature, 378, 355

Naef, D., Mayor, M., Beuzit, J.-L., et al. 2005, in 13th Cambridge Workshop on Cool Stars, Stellar Systems and the Sun, ed. F. Favata, G. A. J. Hussain, \& B. Battrick (ESA Special Publication, Vol. 560) (Noordwijk: ESA), 833

Pont, F., Zucker, S., \& Queloz, D. 2006, MNRAS, 373, 231

Robin, A. C., Reylé, C., Derrière, S., \& Picaud, S. 2003, A\&A, 409, 523

Sackett, P. D. 1999, in NATO ASIC Proc. 532, Planets Outside the Solar System: Theory and Observations, ed. J.-M. Mariotti \& D. Alloin (Boston, MA: Kluwer), 189

Sahu, K. C., Casertano, S., Bond, H. E., et al. 2006, Nature, 443, 534

Schlegel, D. J., Finkbeiner, D. P., \& Davis, M. 1998, ApJ, 500, 525

Tamuz, O., Mazeh, T., \& Zucker, S. 2005, MNRAS, 356, 1466

Tingley, B., \& Sackett, P. D. 2005, ApJ, 627, 1011

van Saders, J. L., \& Gaudi, B. S. 2011, ApJ, 729, 63

Weldrake, D. T. F., \& Bayliss, D. D. R. 2008, AJ, 135, 649

Weldrake, D. T. F., Bayliss, D. D. R., Sackett, P. D., et al. 2008, ApJ, 675, L37

Weldrake, D. T. F., Sackett, P. D., Bridges, T. J., \& Freeman, K. C. 2005, ApJ, 620,1043

Wozniak, P. R. 2000, Acta Astron., 50, 421 